Martin Rössler (2009): Die sozialräumliche Organisation des makassarischen Hauses. In: Elfriede Hermann, Karin Klenke und Michael Dickhardt (Hg.): Form, Macht, Differenz. Motive und Felder ethnologischen Forschens. Göttingen: Universitätsverlag Göttingen, 259-274. Doi: $10.17875 /$ gup $2020-1290$

\title{
Die sozialräumliche Organisation des makassarischen Hauses
}

\author{
Martin Rössler
}

Die folgende Darstellung bezieht sich auf das makassarische Haus im Hochland des südlichen Sulawesi, wobei hier soziale Aspekte der hausräumlichen Struktur im Mittelpunkt stehen sollen. ${ }^{1}$ Von ,dem ' makassarischen Haus zu sprechen, ist im Grunde sehr problematisch. Dies gilt nicht nur angesichts der drastischen Veränderungen in Form und Organisation, die es in jüngster Zeit erfahren hat, sondern vor allem auch im Hinblick auf die Modifikationen, die in zurückliegenden Jahrzehnten vorgenommen wurden. Es wird heute davon ausgegangen, dass die Architektur Süd-Sulawesis viele Gemeinsamkeiten mit derjenigen Süd-Sumatras und mancher Regionen Borneos aufweist. Süd-Sulawesi stellt dabei auch für sich eine spezielle Problematik dar, weil sich hier - neben den in vielerlei Hinsicht identischen Hausformen der Bugis und Makassar - auch die spektakuläre und deutlich abweichende Architektur der Toraja findet, die jedoch anhand neuerer Evidenzen ebenfalls in ein mit der buginesischen und makassarischen Architektur gemeinsames historisches Kontinuum eingebracht werden kann (Pelras 2003:262-265; vgl. Hauser-Schäublin 1985). Die Häuser traditioneller Bauweise erfuhren bis ins 19. Jahrhundert hinein offensichtlich nur geringfügige Modifikationen, darunter das Ersetzen des Bambusfußbodens durch hölzerne Dielen, veränderte Bearbeitungstechniken der hölzernen Pfosten sowie die Einführung von Fenstern und Möbeln. ${ }^{2}$

Wohl in der zweiten Hälfte des 19. Jahrhunderts entstand die bis heute bei Holzhäusern geübte Praxis, die Pfosten nicht mehr in den Erdboden einzulassen, sondern 
auf großen Feldsteinen zu platzieren, was dem gesamten Haus Mobilität verleiht, da es unzerlegt (über ein System aus Bambusrollen) fortbewegt werden kann. Auch die ältere Pfostentechnik wird jedoch bis heute bei einigen makassarischen Gruppen, wie z. B. in Tana Toa, praktiziert (siehe Rössler 1990:319). Im Hinblick auf die jüngere Geschichte ist jedoch vor allem festzuhalten, dass die traditionellen Häuser größtenteils in den 1950er Jahren verschwanden: Aufgrund ihrer als feudalistisch identifizierten und die soziale Hierarchie spiegelnden architektonischen Symbolik wurden die meisten alten Häuser zu jener Zeit von den Rebellen der Darul-Islam-Bewegung niedergebrannt (siehe Pelras 2003:274; Rössler 1997a:278). Ersetzt wurden sie durch Häuser in einem neotraditionellen Stil, der viele Elemente der alten Architektur aufgriff, aber auch schon eine Reihe von Neuerungen aufwies, die seit dem frühen 20. Jahrhundert zunächst von der niederländischen Kolonialverwaltung, später von der indonesischen Regierung propagiert worden waren. Gründe der Hygiene und der Sicherheit, vor allem eine bessere Resistenz gegenüber Feuer, wurden dafür vorgebracht. Darunter fiel insbesondere das Ersetzen der pflanzlichen Materialien für die Dachbedeckung durch Wellblech, das Verlagern von Feuerstellen und Toiletten in Anbauten, das Einbringen von mehreren Schlafkammern - statt traditionell nur einer - sowie das Verbot der Tierhaltung im offenen Raum unter dem Haus (passiringang). ${ }^{3}$

Entscheidend ist jedoch, dass sich die Grundstruktur wie auch die räumliche Organisation des neo-traditionellen Hauses bis auf einige unwesentliche Aspekte nicht von denjenigen des ursprünglichen Hauses unterscheiden. Generell beziehen sich die folgenden Ausführungen auf das neo-traditionelle Haus aus den 1960er Jahren, das bis vor kurzem im Hochland das bei weitem verbreitetste Muster darstellte. ${ }^{4}$ Entscheidende Veränderungen ergaben sich erst im Zuge der rezent drastisch angestiegenen Verbreitung von Ziegelhäusern, auf die ich abschließend eingehen werde. Es muss letztlich auch darauf hingewiesen werden, dass die soziale Schichtung der makassarischen Gesellschaft (siehe Chabot 1996; Röttger-Rössler 1989) architektonische Unterschiede zwischen Häusern des Adels und der Freien impliziert, unter anderem hinsichtlich der erwähnten hierarchiebezogenen Symbolik bestimmter Elemente des Hauses. Darüber hinaus gingen viele Neuerungen in der Hauskonstruktion vom Adel aus und fanden erst später unter den Freien Einzug.

\section{Grundlagen}

Das im Grundriss streng rechteckige makassarische Haus (balla) beruht auf einer H-Rahmen-Konstruktion (siehe Domenig 2003:493) mit vom Boden bis zum Dach durchgehenden Pfosten. Die Wände zwischen den tragenden Rahmenhölzern wurden ursprünglich mit Bambusflechtwerk, später mit Brettern ausgekleidet. Das Giebeldach weist einen geraden, durchgehenden First auf; nach 1960 wurde die ursprünglich konkav abfallende Form der beiden Dachflächen - die mit Wellblech nicht zu konstruieren 
ist - entweder durch gerade Flächen oder durch eine von den Bugis übernommene, stumpfwinklige Form ersetzt (vgl. Abbildung 4). Der Boden des Wohnraumes befindet sich in ca. 1,50 bis (in seltenen Fällen) 2,50 Metern Höhe, die Raumhöhe bewegt sich etwa zwischen 2,00 und 2,50 Metern. Die durchschnittliche Fläche des gesamten Hauses betrug innerhalb meiner Stichprobe von 1990 (N=50) ca. $63 \mathrm{~m}^{2}$, jedoch mit teils erheblichen Abweichungen nach oben wie unten. ${ }^{5}$ Vor der Eingangstür befindet sich auf Höhe des Wohnraumes gewöhnlich eine große, überdachte Veranda (paladang), zu der eine hölzerne Treppe hinaufführt. ${ }^{6}$

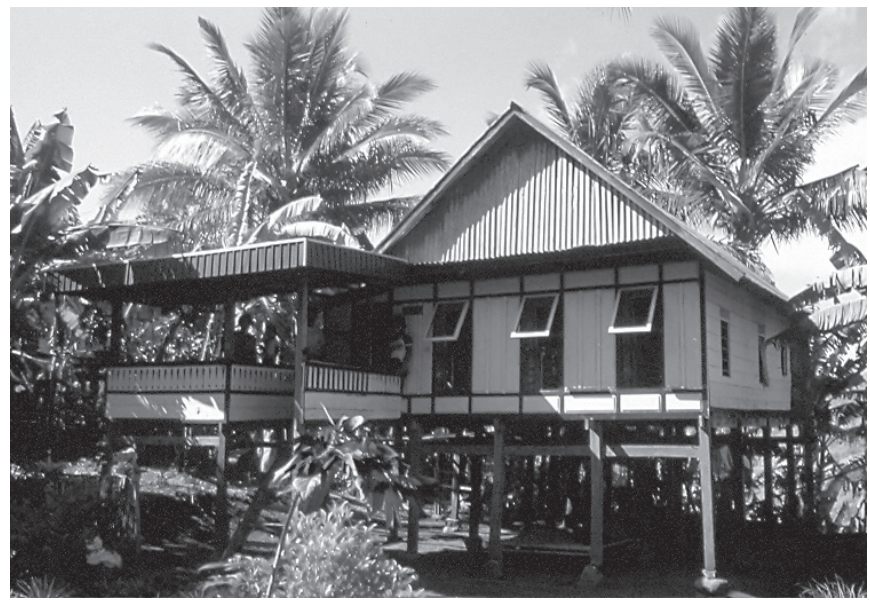

Abbildung 1: Typisches Haus neo-traditioneller Bauart (ca. 1961), mit $34 \mathrm{~m}^{2}$ von unterdurchschnittlicher Größe, jedoch mit sehr hohen Pfosten und großzügiger Veranda. Der nur einteilige Giebel weist auf niederen Deszendenzrang der Besitzer hin.

Foto: Martin Rössler 1984

Das Haus weist eine klare, dreigeteilte vertikale Struktur auf, gebildet aus dem offenen Raum unter dem Fußboden (passiringang), dem Wohnraum (kale balla) und dem Dachspeicher (pammuakkang), die dem vorislamischen Überzeugungssystem zufolge eine Entsprechung der dreigeteilten Struktur des Makrokosmos aus Himmel (boting langi'), Erdoberfläche (lino) und Unterwelt (padatiri) darstellt. Das Unterhaus (früher Raum für Rinder und landwirtschaftliche Geräte) wird dabei konzipiert als Produktionsraum, der Wohnraum als Reproduktions- und Konsumtionsraum und der Reisspeicher unter dem Dach als eine räumliche Vereinigung aus Produktion, Distribution und Konsumtion. ${ }^{7}$

Die Gestaltung des vorderen Dachgiebels richtet sich nach dem sozialen Rang der Hausbewohner. Im Unterschied zu anderen statusdifferenzierenden Details in der Architektur, die mittlerweile verschwunden sind, spielt die Struktur des Giebels nach wie vor eine wesentliche Rolle. Generell ist seine Unterteilung in vertikal geschichtete 
Fächer normativ strikt geregelt: Häuser des Hochadels weisen fünf Fächer, solche des niederen Adels drei, Häuser der Freien zwei oder nur ein Fach auf (siehe Abbildungen 1 und 4). ${ }^{8}$ Ein weiteres Element mit Bezug auf die soziale Schichtung ist mit dem Zugang zum Haus verbunden. Zu Häusern des Hochadels erfolgte er ursprünglich über eine Rampe aus Bambus (sapana) anstelle einer einfachen oder zweigeteilten Treppe, wie sie unter Freien respektive beim niederen Adel in Gebrauch ist. Allein solche Häuser von Nichtadligen, in denen die traditionellen Heiligtümer (kalompoang) aufbewahrt werden, verfügen ebenfalls über eine Rampe.

Die horizontale Struktur des Hauses wird primär in Fächern (lontang oder lasa') gerechnet, die sich aus Längs- und Querreihen der tragenden Pfosten ergeben. Je nach Größe des Hauses findet man meist 4 oder 5 Reihen zu je 4 oder 5 Pfosten, von denen z. B. bei einer $5 \times 5$-Struktur (die jeweils 4 lontang quer und längs ergibt) die zweite und vierte innere Reihe nur vom Erdboden bis zum Fußboden reichen.

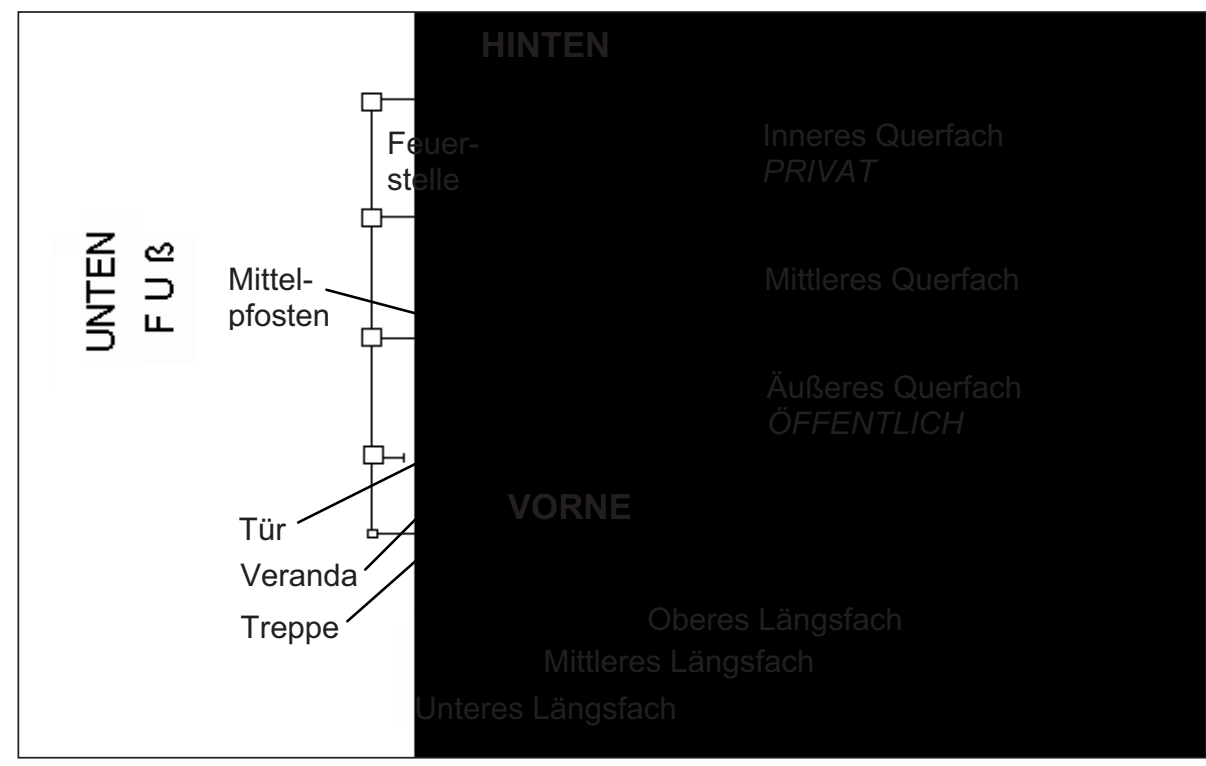

Abbildung 2: Horizontale Struktur des makassarischen Hauses.

Die schematisierte Abbildung 2 geht von einer 4x4 -Struktur aus, die entsprechend eine Ordnung von jeweils drei Quer- und Längsfächern aufweist. Als Orientierungsrichtungen ergeben sich zunächst aus der Außensicht Vorne (dallekang), Hinten (bokoang) und die Seiten (sári), sowie aus der Innensicht die Anordnung der Querfächer von Außen (lontang ipantara') über die Mitte (lontang ritannga) bis nach Innen (lontang ilalang), so dass das Außen mit Vorne sowie das Hinten mit Innen korrespondiert. Eine andere wichtige Ordnung ergibt sich zwischen den Seiten, indem hier ein Übergang vom Fuß zum Kopf, respektive von Unten nach Oben konzeptionalisiert ist. Ent- 
sprechend werden die Längsfächer ebenfalls vom unteren bis zum oberen gerechnet. Von Bedeutung ist dies insofern, als hier ein Bezug zu grundlegenden, im malaiischen Archipel weit verbreiteten räumlichen Orientierungsmustern vorliegt (siehe Rössler 2003:188f., Hauser-Schäublin 2003:65f.), wobei die ideelle Achse Unten-Oben einerseits der Achse Seewärts-Bergwärts entspricht, andererseits aber auch eine hierarchische Dimension im sozialen Sinne impliziert. ${ }^{9}$ Während die Stufung von Unten nach Oben also eine Rangordnung abbildet, ergibt sich in der Richtung von Vorne nach Hinten bzw. von Außen nach Innen ein Übergang vom öffentlichen zum privaten Raum. Im Hinblick auf die Anordnung der Räume ist hier insbesondere die Unterscheidung zwischen dem auch für Gäste offenen großen Wohnraum und der Schlafkammer von Bedeutung, die ebenso wie die Feuerstelle dem privaten Raum zugerechnet wird. Der Ort der Nahrungsaufnahme variiert, je nachdem wer am Essen beteiligt ist. Sind dies allein die Haushaltsmitglieder, so wird meist gleich neben der Feuerstelle gegessen. In Anwesenheit von Nachbarn und Verwandten bewegt sich der Ort der Mahlzeit räumlich geringfügig nach Vorne, bleibt aber dem Unten, dem Fuß des Hauses zugeordnet. Nur bei einem formellen Anlass, an dem Außenstehende und/oder hochrangige Gäste teilnehmen, wird die Mahlzeit in den öffentlichen Bereich des Hauses und gleichzeitig in Richtung Oben verlegt.

Meist am Schnittpunkt zwischen Fuß/Unten und Vorne/Oben befindet sich der Mittelpfosten (benteng tannga). ${ }^{10}$ Er bildet das Zentrum des häuslichen Mikrokosmos und als ,Zentrum des Hauses' (pocci' balla, wörtl. ,Nabel des Hauses') gleichzeitig eine Entsprechung des makrokosmischen Zentrums der Erde (pocci' tana), des Sitzes der Götter. Im Kontext sämtlicher Riten, die innerhalb des Hauses durchgeführt werden, spielt dieser Pfosten eine entscheidende Rolle - ein in austronesischen Gesellschaften weit verbreitetes Phänomen (siehe Fox 1993:21).

Eine überlieferte Regel für die Ausrichtung der Frontseite des Hauses entweder nach Süden oder Osten entstand offensichtlich erst mit der Ankunft des Islam: Von der Tür aus gesehen, weisen die Füße im Schlaf traditionell nach rechts. Damit sie nicht in Richtung auf Mekka gerichtet sind, muss die Hausfront folglich entweder nach Süden (Füße nach Osten) oder nach Osten (Füße nach Norden) hin gelegen sein (siehe Mardanas et al. 1985:73f.). Da sich die weitaus meisten Siedlungen in den letzten Jahrzehnten auf Regierungsanordnung hin am Verlauf von Straßen orientieren mussten, folgt die Ausrichtung der Häuser jedoch heute überwiegend pragmatischen Gesichtspunkten. ${ }^{11}$

\section{Soziale Differenzierung}

Die makassarische Gesellschaft ist geschichtet in Hochadel, niederen Adel, Freie und (ehemalige) Sklaven, wobei die einzelnen Schichten auch in sich differenziert sind. Daneben spielen ebenfalls Unterschiede im Deszendenzrang innerhalb dyadischer 
Beziehungen sowie Differenzierungen nach sozialem Ansehen, Prestige, Alter und hier gesondert behandelt - Geschlecht eine wichtige Rolle.

Innerhalb des häuslichen Raumes spiegeln sich unterschiedliche Aspekte der genannten Hierarchien, so dass Räumlichkeit unmittelbar in deren öffentliche Vermittlung einbezogen wird. Es wurden bereits architektonische Elemente angesprochen, insbesondere die Giebelstruktur, die schichtenspezifische Symbole darstellen. Darüber hinaus ist das Haus als physische Materie jedoch nicht auf direkte Weise entlang hierarchischer Prinzipien strukturiert, so dass etwa seine baulichen Elemente in Entsprechung hierarchischer Ordnungen konzipiert wären. Das Haus besitzt aus der Perspektive sozialer Akteure vielmehr nur ein Potenzial zur räumlichen Umsetzung hierarchischer Prinzipien, das zum Beispiel Positionen innerhalb von Sitzordnungen vorgibt. Aus Gründen, die weiter unten zur Sprache kommen, beschränke ich mich zunächst auf die Positionierung männlicher Individuen im vorderen bzw. äußeren Querfach.

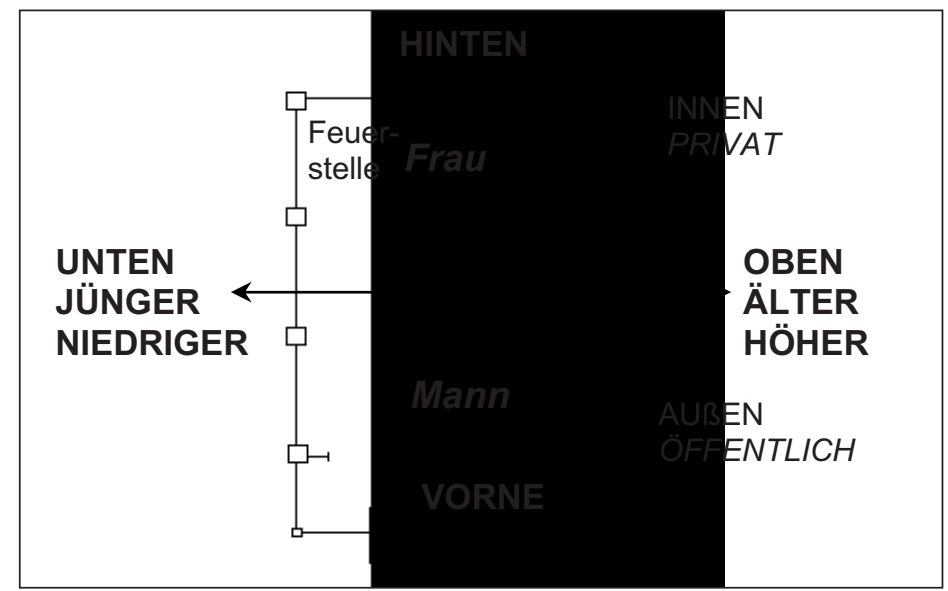

Abbildung 3: Hierarchische und geschlechterbezogene Dimensionen des makassarischen Hauses.

Für die Sitzordnung gilt die Abstufung entlang der Längsfächer bzw. der Ordnung von Unten (irawa) nach Oben (irate), wobei die Haustür grundsätzlich ,unten' positioniert ist. Je höher der individuelle Rang, desto weiter nach ,oben ${ }^{\text {- }}$ desto weiter entfernt von der Tür - die Sitzposition. In der Praxis wird diese einfach anmutende Ordnung dadurch verkompliziert, dass Ränge nicht einfach ,feststehen', sondern in vielen Fällen als ausgehandelt zu verstehen sind, und dass weiterhin Faktoren des Lebensalters und des sozialen Ansehens hinzutreten. Höheres Alter und Ansehen implizieren ebenfalls eine Sitzposition in Richtung nach Oben.

Eine zusätzliche Ordnung nach den Dimensionen Vorne/Hinten bzw. Außen/ Innen wird im Zusammenhang mit der sozialen Differenzierung nicht vorgenommen; diese Dimensionen sind vielmehr im Kontext der Geschlechterbeziehungen von Rele- 
vanz. Neben der horizontalen Ebene ist stets auch die vertikale Dimension des Raumes bedeutsam, und zwar insbesondere in Bezug auf Körperbewegungen und -positionen innerhalb des Hauses: Höherer Rang und höheres Lebensalter erlauben aufrechtere Positionen bei Bewegungen im Haus, während umgekehrt niederer Rang und niederes Lebensalter eine gebeugtere Haltung vorschreiben. Früher - und in rituellen Kontexten bis heute - durften sich Freie in Anwesenheit von Mitgliedern des Hochadels nur auf Knien rutschend fortbewegen. In weniger drastischer Form gelten die Regeln der Körperhaltung nach wie vor unverändert; vor allem darf niemand an einem Sitzenden in aufrechter Haltung vorübergehen. Bei den heute durchweg vorhandenen Sitzmöbeln ${ }^{12}$ ergeben sich dahingehend zusätzliche Akzente: Von jungen und/oder niederrangigen Männern wird erwartet, dass sie sich in Anwesenheit älterer und/oder Ranghöherer im ,unteren' Teil des Raumes auf den Boden niederlassen, anstatt auf Stühlen oder in Sesseln Platz zu nehmen. Es gibt darüber hinaus sozial stark aufgeladene Situationen, etwa wenn ein Adliger im Haus eines Freien zu Besuch ist, in denen junge und niederrangige Männer das Haus überhaupt nicht betreten, sondern sich auf der Veranda oder gar im passiringang aufhalten. Wenn jemand das Haus in einer solchen Situation eigens verlässt, so gilt dies als vorbildliches, Bescheidenheit offenbarendes Verhalten.

In Bezug auf Materialien, Größe und funktionale Ausstattung des Hauses ist weiterhin die erwähnte Dimension individuellen Prestiges bzw. sozialen Ansehens zu berücksichtigen, die in diesem Kontext gegenüber dem Deszendenzrang sogar deutlich in den Vordergrund tritt. Während Prestige und hohes Ansehen nicht notwendigerweise mit dem Rang korreliert sind, ist ihr Zusammenhang mit ökonomischen Faktoren - insbesondere überdurchschnittlichem Wohlstand - häufig augenfällig. Dies äußert sich zum Beispiel in qualitativ besseren Baumaterialien oder teurer Möblierung, wobei das Spektrum der Möglichkeiten in jüngster Zeit deutlich erweitert wurde (siehe unten). Der häusliche Raum wird somit auch in das Aushandeln individuellen Prestiges innerhalb der sozialen Gruppe in direkter Weise einbezogen.

\section{Familie und Haushalt}

Hinsichtlich der Beziehung verwandtschaftlicher und familiärer Kriterien zum häuslichen Raum ist in erster Linie dessen Rolle als materieller und ideeller Rahmen des Haushaltes wichtig. Die Haushaltsmorphologie konstituiert sich vornehmlich anhand verwandtschaftlicher Prinzipien, wobei heute gemeinhin die Kernfamilie (bisweilen ergänzt durch weitere Individuen aus der engeren Verwandtschaft) mit dem Raum des von ihr bewohnten Hauses assoziiert wird. Für den Haushalt (sibatu balla, wörtlich „ein Stück Haus“) gewinnt das Haus als materieller Raum eine direkte sozioökonomische Qualität, indem die Produktions-, Distributions- und Konsumtionseinheit jedes Haushaltes als geschlossenes System konzipiert ist (vgl. Wilk 1994), wobei dies die Kooperation oder den Austausch von Nahrungsmitteln zwischen Haushalten keines- 
wegs ausschließt. Der Raum eines jeden Hauses ist dahingehend einerseits auf ideeller Ebene als in sich abgegrenzt zu verstehen, andererseits jedoch auch hinsichtlich der materiellen Dimension der ,eigenen vier Wände`. So gilt es als zumindest grob unhöflich, ein Haus ohne verbale Anmeldung in Form des lauten Rufes: „Ist der Hausbesitzer da?" zu betreten, es sei denn ein Haus der engsten Verwandtschaft. Erfährt ein männlicher Besucher auf diese Weise, dass nur Frauen im Haus sind, geht er weiter seiner Wege. Für Frauen gilt dies allerdings nicht, wie weiter unten erläutert wird.

Im Hinblick auf den Haushalt hat sich in den letzten Jahren freilich ein deutlicher Wandel gegenüber den Verhältnissen von vor noch etwa einem halben Jahrhundert vollzogen, da Haushalte früher ganz andere Strukturen aufwiesen: In den damals deutlich kleineren Häusern wohnten erweiterte Familien mit nicht selten 10-15 Personen zusammen (gegenüber einer mittleren Haushaltsgröße von knapp 5 Personen 1990 und 4,2 Personen 2005) ${ }^{13}$, wobei die einzelnen Kernfamilien (bija) sich zwar gewöhnlich die Schlafkammer teilten, jedoch jeweils eigene Feuerstellen hatten und voneinander getrennte Konsumtionseinheiten bildeten (siehe Rössler 1997b:346f.). Von den früheren Verhältnissen, mit deutlich weniger Wohnraum pro Familie, wurde mir berichtet, dass gezielte Abgrenzungen zwischen den in einem Haus zusammen lebenden Kernfamilien die Regel waren und generell ein bedeutendes Konfliktpotenzial vor allem bezüglich der Zuweisung von Nahrungsmitteln bestand. Da hier ein Haus mehrere Familien und Feuerstellen umfasste, galten letztere ursprünglich als soziale Zentren der einzelnen Haushalte und gleichzeitig als Indizes ihrer Geschlossenheit (vgl. Carsten 1997, Sather 1993).

\section{Geschlechterverhältnis und Lebenszyklus}

In diesem Kontext kommt der geschlechterbezogene Aspekt hinzu. Zunächst ist es eine grundlegende Tatsache, dass sich Frauen auffällig freier in fremden Häusern wie auch generell in der Öffentlichkeit bewegen können als Männer (siehe Röttger-Rössler 1994). Der häusliche Raum ist prinzipiell aufgeteilt zwischen einem exklusiven (,privaten ${ }^{c}$ ) und einem inklusiven (,öffentlichen') Bereich, wobei die Übergangszone kontextabhängig leicht verschiebbar ist, wie unter anderem in rituellen Kontexten, in denen allein die Menge der Anwesenden eine entsprechende Trennung häufig nicht erlaubt. In jedem Fall ist jedoch die Aufenthaltszone für Männer in fremden Häusern strikt auf den unzweifelhaft inklusiven Raum beschränkt, das heißt, auf das äußere/vordere Querfach (siehe Abbildung 3), innerhalb dessen dann die oben beschriebenen Regeln der Raumnutzung in den Dimensionen von Unten nach Oben gelten. Dass der Raum der Männer mit dem Außen und der Öffentlichkeit assoziiert ist, steht also in einem gewissen Widerspruch zu der Tatsache, dass Männer in ihren Raumbewegungen im Unterschied zu Frauen erheblichen Restriktionen unterlegen sind. Zwar ist der weibliche Bereich grundsätzlich mit dem inneren/hinteren Raum des Hauses assoziiert, doch 
steht Frauen - auch in anderen Häusern als ihren eigenen - grundsätzlich der gesamte Raum offen. Dahingehend ergibt sich eine deutliche Steigerung im Verlauf des Lebens: Während sich junge Mädchen und unverheiratete Frauen speziell im Falle männlicher Besucher in den privaten Bereich zurückziehen, verliert dieser ,weibliche Raum' des Hauses für ältere bis alte Frauen seine Bedeutung als sozialräumlicher Fixpunkt in zunehmendem Maße.

Hierzu einige Erläuterungen: Im Schnittbereich zwischen den Quer- und Längsfächern des Innen/Hinten und Unten befindet sich die Feuerstelle, während in der gleichen inneren Querachse, nur am Kopfende (,Oben') des Hauses traditionell die Schlafkammer liegt, der Raum der Reproduktion und der Rekreation. Dieser exklusive Bereich, bzw. die Räume der Nahrungszubereitung und der Reproduktion sind unmittelbar mit dem Weiblichen assoziiert. Obwohl heute meist zwei oder gar drei Schlafkammern üblich sind, hat sich ihre grundsätzliche Orientierung im Raum des Oben und des Inneren nicht geändert. Die auffallende Parallele von Innen/Privat/Weiblich ist im gesamten austronesischen Sprachraum zu finden (Fox 1993:16), im hier diskutierten Fall jedoch auch vor dem Hintergrund der ursprünglich rigiden Geschlechtertrennung der makassarischen Gesellschaft zu verstehen. ${ }^{14}$ Der hintere und innere, intimste Bereich des Hauses soll und muss, wie man heute nach wie vor sagt, in erster Linie die Frauen schützen. Dies galt bis vor einiger Zeit vor allem für die unverheirateten Mädchen, die das Haus prinzipiell nicht ohne Aufsicht verließen und die sich bei Anwesenheit männlicher Gäste sofort in die privaten Bereiche zurückzogen. Obwohl dies heute längst nicht mehr so strikt gehandhabt wird, besteht das ideelle Kontinuum zwischen Frau/ Innen/Hinten auf der einen Seite sowie Mann/Außen/Vorne auf der anderen unverändert fort. Auch wenn die bauliche Konstruktion des Innenraumes vom traditionellen Schema heute gewöhnlich abweicht, insbesondere in Gestalt mehrerer Kammern und größerer Küchenräume, so wird durch das Einziehen zusätzlicher Sperrholzwände die Grenze zwischen ,Privat' und ,Öffentlich' immer noch eindeutig markiert.

Auf einer allgemeineren Ebene wird die Beziehung zwischen dem häuslichen Raum und lokalen Geschlechterkonzeptionen überwiegend über geschlechtsspezifische Aktivitäten und Rollenzuweisungen hergestellt. Von Bedeutung sind hinsichtlich der Aktivitäten in erster Linie produktive, reproduktive sowie soziale Aktivitäten. Die produktiven und reproduktiven Aktivitäten der Frau sind generell mit dem Raum des Hauses im Allgemeinen bzw. mit der Feuerstelle und der Schlafkammer im Besonderen assoziiert. Dahingehend ist auch zu betonen, dass die Frau nicht nur Besitzerin des Hauses im rechtlichen Sinne ist - insofern als es ihr bei einer Ehescheidung zufällt -, sondern auch als eine Art Garant für den Fortbestand des Hauses und des Haushaltes in sozialer wie in ökonomischer Sicht aufgefasst wird (vgl. Waterson 1993:227). Der Übergang von Aktivitäten zu Rollenzuweisungen ist vor diesem Hintergrund fließend. Die weibliche Rolle wird konzeptionalisiert als auf das Innen, auf das Geschlossene, auf den exklusiven Raum bezogen. Allerdings verfügt die Frau, wie oben angedeutet, über die Möglichkeit, sich frei zwischen diesen exklusiven wie auch zwischen inklusiven 
Räumen nicht nur im eigenen, sondern auch in fremden Häusern zu bewegen. Hingegen ist die Bewegung des Mannes von einem inklusiveren Raum in einen anderen (auch des Verwandten, des Nachbarn) deutlich formalisierter, während ihm die Nutzung fremder exklusiver Räume normativ absolut verschlossen ist.

In diesem Zusammenhang ist abermals der Aspekt der Sitzordnungen zu erwähnen. Prinzipiell besteht in dieser Hinsicht zwar die Tendenz, dass Frauen mit dem ,Unten' bzw. mit der Übergangszone zwischen dem inklusiven und dem exklusiven Bereich (Abbildung 3) assoziiert sind. Diese Tendenz äußert sich jedoch in der Praxis oft nur undeutlich, da insbesondere der Faktor des sozialen Ranges hier schnell zu Verschiebungen führen kann. Eine adlige Frau wird in einem Raum gemeinsam mit Freien normativ im sozusagen ,extremen' Schnittpunkt von Öffentlich/Oben/Vorne unter den Männern positioniert. Betrachtet man das normative Ideal der nach Geschlecht separierten Gruppen, so ist die Sitzordnung innerhalb der Frauen jedoch generell deutlich weniger strikt als innerhalb der Männer. Soziale Rangunterschiede, selbst zwischen adligen und nichtadligen Frauen, spielen im Hinblick auf Sitzpositionen kaum eine Rolle. Es ist sogar fast selbstverständlich, dass sich eine Adlige als Gast im Haus von Freien in die Küche begibt, um dort zu helfen. Die beschriebenen normativen Muster sozialer Positionierungen im geschlechtsspezifischen Sinne gelten im häuslichen Raum folglich nur für die öffentliche und äußere - und ,männliche - Querachse, während sie in der privaten und inneren - und ,weiblichen - eher neutralisiert werden.

Die Einbeziehung des menschlichen Lebenszyklus' vermittelt ergänzende Hinweise auf die Zusammenhänge zwischen häuslichem Raum und sozialen Faktoren. Das menschliche Leben beginnt an der Schnittstelle der Achsen des Privaten/Inneren und des Oben, wo Kinder geboren und auch den ersten Übergangsriten unterzogen werden. Insbesondere für die Mädchen blieb dieser Raum, wie gesehen, bis zur Heirat traditionell der häusliche Hauptorientierungspunkt. Die Heirat markiert eine Umorientierung vom Innen zum Außen: Im Verlauf der Hochzeitsriten wechseln Braut und Bräutigam mehrmals zwischen ihren Elternhäusern hin und her, was eine hochgradig öffentliche Raumbewegung darstellt. Hinzu kommt der Sachverhalt, dass die junge Ehefrau von nun an in die Lage versetzt ist, sich in den öffentlichen Räumen aller Häuser zu bewegen. Es findet also speziell für die Braut ein symbolischer Transfer aus der Abgeschiedenheit der Kammer hin zur diametral entgegengesetzten Tür des Hauses, zum Weg in die Öffentlichkeit außerhalb des Hauses statt.

Im Alter werden die Bewegungen und Aktivitäten wieder mehr und mehr ins Haus zurückverlagert. Ich habe in vielen Fällen erlebt, dass sich alte Leute regelrecht schämen, sich mit Hilfe der Kinder und Enkel mühsam vor das Haus zu begeben, um weiterhin am öffentlichen Leben teilzunehmen. Von alten Menschen werden leichtere Arbeiten im mittleren Querfach verrichtet, bis ihr Aufenthaltsort gegen Ende des Lebens schließlich wieder in die innere Querachse zurückgeführt wird, und zwar generell auf ein Lager in der Nähe der Feuerstelle, nicht in die Kammer, in der die Kinder und Enkel schlafen (vgl. ähnlich zu Minangkabau, Ng 1993:124-8). Auf diese Weise wird eine 
Analogie zwischen dem physischen Prozess des Alterns und einer räumlichen Rückzugsbewegung innerhalb des Hauses vollzogen. Das Leben endet neben dem Ort, an dem es begonnen hatte: neben der Kammer, die für die Reproduktion und den Beginn neuen Lebens reserviert bleibt.

\section{Rezenter Wandel}

Um 1990 herum betrug der Anteil der neo-traditionellen Häuser, wie sie hier als Grundlage der Darstellung dienten, innerhalb der von mir untersuchten Gemeinschaft noch 76\%. Im Jahre 2005 war er bereits auf 36\% gefallen. Die meisten hölzernen Häuser waren durch Ziegelbauten ersetzt worden, die zum einen vom Material her heute wesentlich billiger als Holzhäuser zu errichten sind, zum anderen jedoch auch den geschätzten Aspekt der ,Modernität' implizieren..$^{15}$ Es sollte in diesem Zusammenhang erwähnt werden, dass im Unterschied zu vielen Aspekten der Landschaft (siehe Rössler 2009:306f.) makassarische Häuser kaum in einem Zusammenhang mit historischen Erinnerungen stehen und nur in Ausnahmefällen eine Rolle als Fokus sozialer Beziehungen oder Ursprung von Deszendenzgruppen spielen, wie dies ansonsten in Indonesien häufig zu finden ist (Fox 1993:16f., Waterson 1990) ${ }^{16}$ Der Abriss eines Hauses ist hier kein Akt, dem eine besondere Bedeutung beigemessen würde.

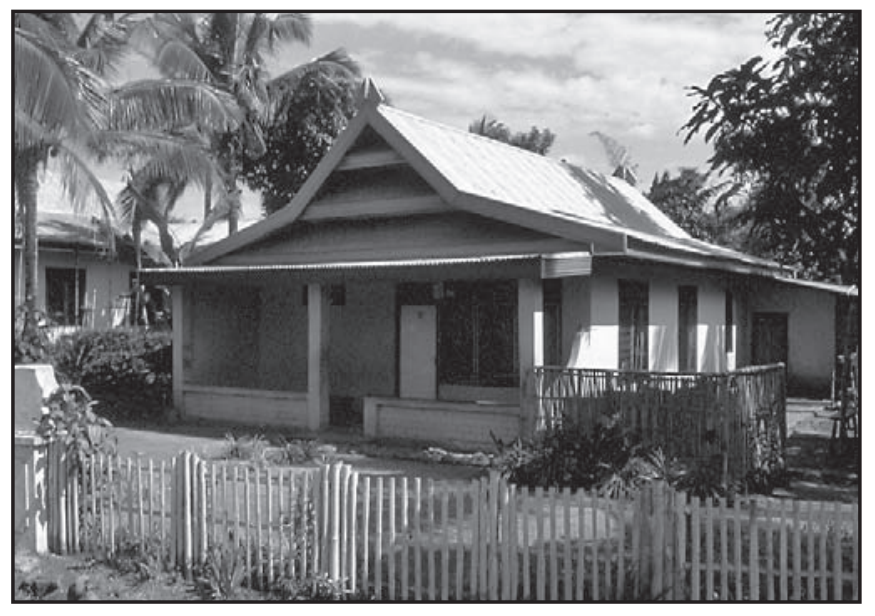

Abbildung 4: Ziegelhaus von $1987\left(66 \mathrm{~m}^{2}\right)$. Das gewinkelte Dach nach buginesischem Vorbild und der dreigestufte Giebel sind die einzigen Elemente, die von der früheren Architektur übernommen wurden. (Foto: Martin Rössler 1989) 
Es offenbart sich heute gegenüber früher eine deutliche Heterogenität in Bauweise und Ausstattung der Häuser, die mit einer zunehmenden ökonomischen Polarisierung zwischen wohlhabenden und verarmten, landlosen Haushalten einhergeht. ${ }^{17}$ Im hier diskutierten Kontext ist jedoch die veränderte Struktur und Organisation insbesondere der Ziegelbauten relevant. Im Unterschied zur Konzeption des neo-traditionellen Hauses, die wie erwähnt nur unwesentlich von derjenigen des traditionellen abwich, ergeben sich nun durch neue Bauprinzipien drastische Veränderungen, die zahlreiche Konsequenzen auch in Bezug auf das menschliche Verhalten nach sich ziehen (siehe auch Pelras 2003:276f.). Die ebenerdig errichteten Ziegelhäuser weisen in der vertikalen wie in der horizontalen Dimension keine kosmologischen Bezüge auf und entsprechen in der Regel nicht den Orientierungsmustern, wie sie für die meisten der hier angesprochenen Punkte grundlegend waren. Hierarchien, Prinzipien der geschlechtsund altersspezifischen Ordnung von Raumaufteilungen und -bewegungen können nicht mehr räumlich umgesetzt werden. Die wenigen ursprünglichen symbolischen Elemente, die übernommen wurden, sind aus dem kulturellen Kontext gerissen. Bei den Giebelfächern kommt hinzu, dass zunehmend mehr Nichtadlige ein ihrem Stand ,unangemessenes' Rangsymbol verwenden - wie auch im Falle des Hauses in Abbildung 4 geschehen.

Hinsichtlich der sozioökonomischen Differenzierung ergeben sich seit kurzer Zeit durch die Verfügbarkeit von Fliesenfußböden, WC, Kücheneinrichtungen nach westlichem Vorbild und elektrischen Geräten neue Möglichkeiten zum Aushandeln von Prestige, wobei die Nachfragetrends in immer rascherer Folge von Fernsehgeräten über DVD-Spieler jüngst hin zu Kühlschränken verlaufen sind. ${ }^{18}$ In vielen Fällen eröffnete sich dahingehend eine neue Dimension in der öffentlichen Präsentation sozialer Differenzierung.

Auch deutlich negative Aspekte sind zu bedenken. Die neue Architektur ist klimatisch nicht angepasst: Infolge der fehlenden Luftzirkulation heizen sich Ziegelhäuser in der Trockenzeit extrem auf, während in der Regenzeit feuchte Kälte und Schimmelbildung die Regel sind und die häufigen Überflutungen massive Bauschäden zur Folge haben. Schließlich ist es im Unterschied zur Holzbauweise auch nicht länger möglich, ein Haus entweder unzerlegt fortzubewegen, oder es zu zerlegen und an einem anderen Ort wieder zu errichten. Dieser Punkt hat insbesondere für das Erb- und Scheidungsrecht Konsequenzen: Ziegelhäuser bedeuten immer eine feste Bindung an eine Lokalität, die dem früheren Muster der Mobilität - samt Haus - im Erb- oder Scheidungsfalle widerspricht.

Die neue Architektur ist nicht mehr in der Lage, soziale Ordnungen räumlich auf die gleiche Weise wie in den traditionellen Häusern umzusetzen, und das komplexe Wissenssystem, wie es mit dem traditionellen Haus verbunden war, verblasst insbesondere bei den jüngeren Generationen äußerst rasch. Bemerkenswert ist allerdings, dass im Unterschied zu den neuen, als „,modern“ begriffenen Baumaterialien und zur Ausstattung der Häuser mit neuen Konsumtionsgütern, die bedeutende Investitionen erfor- 
dern und Statusindikatoren darstellen, die neue räumliche Struktur des Hauses von den Einheimischen in keiner Weise thematisiert wird. Die ältere Generation beklagt ebenso wenig verloren gegangene räumliche Ordnungen, etwa in Gestalt von Sitzpositionen, wie die Jüngeren in dieser Hinsicht neue Autonomiespielräume artikulieren. Die überlieferten, generations- wie rangspezifischen Normen und Verhaltensmuster spielen im Alltag nach wie vor eine große Rolle. Dass sie innerhalb des Hauses nicht mehr in direkter Weise räumlich umgesetzt werden, spiegelt folglich keine grundsätzlichen sozialen Veränderungen wider. Architektonischer und gesellschaftlicher Wandel stehen nur in insofern in einer Beziehung zueinander, als das Bild des modernen, nach westlichem Vorbild gestalteten Ziegelhauses in übergreifender Hinsicht populär geworden ist. Normen des alltäglichen sozialen Umgangs innerhalb der dörflichen Gemeinschaft sind dadurch nicht betroffen. Weder sind folglich die Modifikationen der Hauskonstruktion als Konsequenz sozialen Wandels auf der Mikroebene aufzufassen, noch wäre es zulässig, sie als einen unmittelbaren Ausgangspunkt für eine künftige Auflösung überlieferter gesellschaftlicher Ordnungen und Umgangsformen zu betrachten.

\section{Anmerkungen}

1 Die Darstellung basiert auf einer Reihe von Feldaufenthalten seit 1982. - Alle im Text kursiv gesetzten Begriffe entstammen dem Makassarischen.

2 Die Möblierung entstand wahrscheinlich um 1600 unter portugiesischem Einfluss; makassarische Begriffe wie kadera (Stuhl), mejang (Tisch), lamari (Schrank) oder jandela (Fenster) sind - ähnlich wie im Malaiischen - portugiesischen Ursprungs.

3 Das Verschwinden der Dachspeicher (pammuakkang), wie es Pelras (2003:275) für die buginesischen Regionen beschreibt, wurde ebenfalls aus hygienischen Gründen forciert, um Ratten und Ungeziefer von den Häusern fernzuhalten. Es hängt aber dort ursächlich mit der Einführung neuer Reissorten zusammen, die nicht mehr in Bündeln auf dem Speicher gelagert werden können. Da die alten Sorten im makassarischen Hochland bis heute eine wichtige Rolle spielen, gibt es hier in den neo-traditionellen Holzhäusern und selbst in vielen Ziegelhäusern nach wie vor Dachspeicher (siehe Rössler 1997b).

4 Ich hatte in den 1980er Jahren noch die Gelegenheit, einige wenige Häuser aus der Vorkriegszeit zu dokumentieren, die jedoch kurze Zeit später abgerissen wurden (siehe Rössler 1997b:Abbildung 35). Ein auffälliges Element, das in den neo-traditionellen Häusern nicht übernommen wurde, war eine Art Flur (sonrong) von der Tür bis zur rückwärtigen Feuerstelle, der gegenüber dem Boden des Wohnraumes um ca. $30 \mathrm{~cm}$ abgesenkt war.

5 Aus Platzgründen gehe ich auf technische Aspekte und Baumaterialien nicht näher ein. Siehe hierzu Pelras 1975 und 2003 sowie Mardanas et al. 1985, wobei letztere auch die TorajaArchitektur detailliert darstellen.

6 Fehlt die Veranda, für die erhebliche Mengen an Baumaterial benötigt werden, so deutet dies auf ein schmales Haushaltsbudget hin. Generell erlaubt es die Konstruktion des Hauses aber, stets etwas zu erweitern oder anzubauen, so dass manche Exemplare sogar ihre ursprünglich rechteckige Grundstruktur völlig verloren haben.

7 Ebenso wie jede Ebene des Makrokosmos von bestimmten Göttern und Geistwesen beherrscht wird, so wird jede Schicht des Hauses mit bestimmten übernatürlichen Mächten 
assoziiert. Auch auf diese Aspekte einschließlich ihrer rituellen Umsetzung kann ich hier nicht eingehen. - Nicht nur in stadtnahen Siedlungen ist man mehr und mehr dazu übergegangen, den passiringang mit Flechtwänden oder gar Ziegelmauern zu umgeben, um zusätzliche geschlossene Räume zu gewinnen, die durchaus auch als Wohnraum dienen können.

8 Letzteres in Abhängigkeit davon, ob es sich um das Substratum der tu baji' (aus privilegierten kognatischen Deszendenzgruppen) oder der tu samara' handelt. Die Einteilungen unterliegen regionalen Variationen.

9 Konzeptionen von Reinheit gegenüber Unreinheit, wie sie im hinduistischen Bali mit einer identischen Orientierungsachse verbunden werden (Hauser-Schäublin 2003:66,80), finden sich in Sulawesi nicht.

$10 \mathrm{Im}$ buginesischen Haus wird die Position des Mittelpfostens abweichend und offensichtlich präziser definiert (siehe Pelras 2003:257,270). Im makassarischen Hochland gibt es demgegenüber von Haus zu Haus oft erhebliche Abweichungen in Bezug auf die Positionierung des benteng tannga. $\mathrm{Ob}$ dies in früheren Zeiten anders war, konnte ich nicht ermitteln.

11 Ähnliches gilt auch für die Anordnung der recht luftigen (weil mit gesplittetem Bambus verkleideten) Küchen, die weniger nach ,traditioneller Vorschrift' angelegt werden, sondern in der Regel auf der östlichen Seite, weil sie hier den stürmischen Regengüssen des Westmonsuns weniger Angriffsfläche bieten.

12 Hier muss angemerkt werden, dass Sitzmöbel in Häusern mit Heiligtümern fehlen und in allen Häusern bei rituellen Anlässen grundsätzlich fortgeräumt werden.

13 Die nach 1990 weiter gesunkene Haushaltsgröße ergab sich in jüngster Zeit hauptsächlich durch eine verstärkte Abwanderung jüngerer Leute.

$14 \mathrm{Da}$ sich diesbezüglich heute viele Änderungen ergeben haben, ist die Darstellung Chabots (1996) mit Daten aus den 1930er Jahren über die früheren Verhältnisse aufschlussreich.

15 Für ein Holzhaus neo-traditioneller Bauweise wird eine Haltbarkeitsdauer von etwa 50 Jahren angegeben, da selbst die Rahmenkonstruktion dann erhebliche Verfallserscheinungen aufweist. Dies steht in deutlichem Kontrast zu den alten Blockbauten der Sa'dan-Toraja, deren Lebensdauer - auch aufgrund der grundlegend anderen Konstruktionsweise - ein Vielfaches beträgt (Hauser-Schäublin 1985).

16 Entsprechendes gilt mit starken Einschränkungen allenfalls für das Haus der politischen Führung, in dem das Heiligtum aufbewahrt wird. Auch dieses wird jedoch nicht etwa in größeren Zeitabständen erneuert, wie die tongkonan der Toraja (Hauser-Schäublin 1985, Waterson 1993), sondern - wenn es nach Jahrzehnten nicht mehr bewohnbar ist - dem Verfall überlassen. Traditionell durfte es nicht abgerissen werden; auch dieser Regel wird aber heute nicht mehr entsprochen.

17 Es hat sich hier innerhalb weniger Jahre eine den javanischen Verhältnissen ähnliche Entwicklung ergeben, die um 1990 allenfalls in Ansätzen erkennbar war (siehe Rössler 1997b).

18 Im Hinblick auf diese Aspekte war die 1993 erfolgte Elektrifizierung der Region ein entscheidender Schritt.

\section{Literatur}

Carsten, Janet (1997) The Heat of the Hearth. The Process of Kinship in a Malay Fishing Community. Oxford: Oxford University Press. 
Chabot, H. Th. (1996 [1950]) Kinship, Status and Gender in South Celebes. Leiden: KITLV Press.

Domenig, Gaudenz (2003) Glossary of Technical Terms. In: Reimar Schefold et al. (Hg.): Indonesian Houses Vol 1: Tradition and Transformation in Vernacular Architecture, S. 483-514. Leiden: KITLV Press.

Fox, James J. (1993) Comparative Perspectives on Austronesian Houses: An Introductory Essay. In: James J. Fox (Hg.): Inside Austronesian Houses: Perspectives on Domestic Designs for Living, S. 1-28. Canberra: ANU.

Hauser-Schäublin, Brigitta (1985) Blockbauten der Sa'dan Toraja. Materialien zur Geschichte der Toraja aufgrund von früheren Hausformen. In: Wolfgang Marschall (Hg.): Der grosse Archipel. Schweizer ethnologische Forschungen in Indonesien, S. 59-82. Bern: Schweizerische Ethnologische Gesellschaft.

Hauser-Schäublin, Brigitta (2003) Raum, Ritual und Gesellschaft. Religiöse Zentren und sozio-religiöse Verdichtungen im Ritual. In: Brigitta Hauser-Schäublin und Michael Dickhardt (Hg.): Kulturelle Räume - räumliche Kultur. Zur Neubestimmung des Verbältnisses zweier fundamentaler Kategorien menschlicher Praxis, S. 43-87. Münster: Lit.

Mardanas, Izarwisma et al. (1985) Arsitektur tradisional daerah Sulawesi Selatan. Ujung Pandang: Departemen Pendidikan dan Kebudayaan.

Ng, Cecilia (1993) Raising the House Post and Feeding the Husband-Givers: The Spatial Categories of Social Reproduction Among the Minangkabau. In: James J. Fox (Hg.): Inside Austronesian Houses: Perspectives on Domestic Designs for Living, S. 116-139. Canberra: ANU.

Pelras, Christian (1975) La maison Bugis: Formes, structures et fonctions. Asie du SudEst et du Monde Insulindien 6(2):61-100.

Pelras, Christian (2003) Bugis and Makassar Houses; Variation and Evolution. In: Reimar Schefold et al. (Hg.): Indonesian Houses Vol 1; Tradition and Transformation in Vernacular Architecture, S. 251-84. Leiden: KITLV Press.

Rössler, Martin (1990) Striving for Modesty: Fundamentals of the Religion and Social Organization among the Makassarese Patuntung. Bijdragen tot de Taal, Land-en Volkenkunde 146(2-3):289-324.

Rössler, Martin (1997a) Islamization and the Reshaping of Identities in Rural South Sulawesi. In: Robert Hefner und Patricia Horvatich (Hg.): Islam in an Era of Nation-States: Politics and Religious Renewal in Muslim Southeast Asia, S. 275-306. Honolulu: University of Hawaii i Press. 
Rössler, Martin (1997b) Der Lohn der Mühe; Kulturelle Dimensionen von ,Wert' und , Arbeit' im Kontext ökonomischer Transformation in Süd-Sulawesi, Indonesien. Münster: Lit.

Rössler, Martin (2003) Landkonflikt und politische Räumlichkeit: Die Lokalisierung von Identität und Widerstand in der nationalen Krise Indonesiens. In: Brigitta Hauser-Schäublin und Michael Dickhardt (Hg.): Kulturelle Räume - räumliche Kultur. Zur Neubestimmung des Verbältnisses zweier fundamentaler Kategorien menschlicher Praxis, S. 171-220. Münster: Lit.

Rössler, Martin (2009) The Anthropological Study of Landscape. In: Michael Bollig und Olaf Bubenzer (Hg.): African Landscapes: Interdisciplinary Approaches, S. 297-325. New York: Springer.

Röttger-Rössler, Birgitt (1989) Rang und Ansehen bei den Makassar von Gowa (SüdSulawesi/Indonesien). Berlin: Reimer.

Röttger-Rössler, Birgitt (1994) „Frauen sind freier..... Geschlechterrollenwechsel in einer indonesischen Gesellschaft. Kea 7:87-108.

Sather, Clifford (1993) Posts, Hearths and Thresholds: The Iban Longhouse as a Ritual Structure. In: James J. Fox (Hg.): Inside Austronesian Houses: Perspectives on Domestic Designs for Living, S. 65-115. Canberra: ANU.

Waterson, Roxana (1990) The Living House. An Anthropology of Architecture in SouthEast Asia. Singapore: Oxford University Press.

Waterson, Roxana (1993) Houses and the Built Environment in Island South-East Asia: Tracing Some Shared Themes in the Use of Space. In: James J. Fox (Hg.): Inside Austronesian Houses: Perspectives on Domestic Designs for Living, S. 221-235. Canberra: ANU.

Wilk, Richard R. (1994) Inside the Economic Institution: Modelling Household Budget Structures. In: James M. Acheson (Hg.): Anthropology and Institutional Economics, S. 365-390. Lanham: University Press of America. 